\title{
The analysis of integration test result for ATM switching systems
}

\author{
Seung-kook Cheong \\ Kyeong-ho Lee, Taeg-won Jeong
}

Electronics and Telecommunications Research Institute

161 Kajong-Dong, Yusong-Gu, Taejon, 305-600, KOREA

FAX : +82-42-860-5410

$<$ e-mail address $>$

skjeong@nice.etri.re.kr/khlee@nice.etri.re.kr/twjeong@nice.etri.re.kr

\begin{abstract}
This paper presents a new analysis method of integration test result for ATM switching systems. The new analysis method, called XTAM(eXchange system Test result Analysis Method), will provide numerical parameters from test results which are vague, qualitative and descriptive. The analysis method is based on Fuzzy theory. This method has been successfully applied to the ATM switching system which is under development by ETRI in Korea.
\end{abstract}

Keywords

Switching System, Fuzzy Set, Test Analysis, XTAM, Integration Test, Regression Test

\section{INTRODUCTION}

System integration test is one of the most important activities during the development of complex systems such as ATM, N-ISDN, PSTN switching systems.[1] The test is valuable only if the result is correctly analyzed and notified to the related developers. Therefore, correct analysis of test results is indispensable for the improvement of system. Usually test results are used just as data for debugging rather than as data for influences of the errors found during the test and for the consideration needed to implement more functions.[2-3] 
The limited use of test results can be considered as the waste of resources.

In this paper a new analysis is proposed to give quantitative description about the influences of faults on the system, extent of implementation for each function, stability of the system, etc. This analysis is based on the Fuzzy theory.

\section{ANALYSIS METHODOLOGY}

System integration tests are carried out for the total system rather than for each part of the system during the development of system. Errors, caused by faults in hardware, software, firmware, or combination of several factors, are debugged according to the information found during system tests[3].

The test of systems and the analysis of test results are usually done at the source level[4-7]. Hence there are not much study on the analysis of test results at system level. In our approach test results are analyzed to provide various parameters which are helpful for various R\&D stages. The analysis system, called XTAM(eXchange system Test result Analysis Method) has the following characteristics :

- Define probable(expected) errors

- Integration and analysis of errors found during tests

- Numerical representation of errors which are descriptive and qualitative

- Transform numerical representation value into possibility distribution function

- Extract raw data needed for the analysis

- Analyze and find distribution for each error type.

At first, probable errors are defined and categorized. Probable errors depend on R\&D environments. In this paper, 7 types of errors are defined as in Table 1. The error types should be defined such that an error may be contained in a unique error type.

Table 1 Types of probable errors

\begin{tabular}{ccc}
\hline Error Type & Function & Cause of error \\
\hline a & Errors by Hardware Failure & Hardware malfunction \\
b & Firmware Logic Error & Firmware \\
c & Operating System Error & Operating System \\
d & Data Base Error & DBMS or incorrect data \\
e & Administration Application Error & Administration software \\
f & Maintenance Application Error & Maintenance software \\
g & Call Processing Application Error & Call processing software \\
\hline
\end{tabular}

Next stage is detailed classification and numerical representation of found errors by system testing execution. This should be done carefully by system engineering experts with abundant 
experiment, since the numerical value is the raw data for analysis. Basically the numerical representation procedure of errors which are descriptive and qualitative are based on discussion with system function developers. Also, found errors during system tests may belong to several predefined error types(refer. Table 1). The numerical value is defined as the membership function of fuzzy set. The following describes how to transform descriptive and qualitative data into numerical value(membership function in fuzzy set).

- Each found error is considered as independent and must decide probable error types. For example, if found error belongs error types $a, b$, and $e$, then the membership function of each error type $(a, b$, and $e)$ will be determined some value from 0 to $1\left(0 \leq \mu_{x}(x) \leq 1\right)$.

- The membership function for each determined error type takes any value from 0 to 1 . The value is determined according to the following relation. $\mu_{x}(x)=0$ no have relation, $0<\mu_{x}(x)<0.3$ have weak relation, $0.3 \leq \mu_{x}(x)<0.7$ have middle relation, and $0.7 \leq \mu_{x}(x) \leq 1$ have strong relation with predefined error types.

- The exact value of this membership function is determined as the mean by several experts. For example, if 3 experts assign $0.3,0.4,0.2$ for error respectively, it is decided to 0.3 .

After the numerical representation of errors, errors are categorized as in Table 2. using the possibility distribution function in fuzzy theory. Raw data, possibility distribution function, are acquired from membership function of fuzzy set.

Table 2 Possibility distribution of found errors

\begin{tabular}{ccccccccc}
\hline Found & \multicolumn{7}{c}{ Error Types' } \\
\cline { 2 - 8 } Error & $a$ & $b$ & $c$ & $d$ & $e$ & $f$ & $g$ & Remark \\
\hline $\mathrm{v}=\mathrm{E}_{1}$ & $\pi_{\mathrm{v}}(a)$ & $\pi_{\mathrm{v}}(b)$ & $\pi_{\mathrm{v}}(c)$ & $\pi_{\mathrm{v}}(d)$ & $\pi_{\mathrm{v}}(e)$ & $\pi_{\mathrm{v}}(f)$ & $\pi_{\mathrm{v}}(g)$ & Error types are defined in Table 1.
\end{tabular}

After the preparation of possibility distribution function, errors are analyzed to give useful parameters using the data in possibility distribution function. Parameters are defined and found according to the following definitions.

$$
|I|=\sum_{y \in Y} \mu_{l}(y),|T|=\sum_{x \in X} \mu_{r}(x),\|I\|=\frac{|I|}{|Y|},\|T\|=\frac{|T|}{|X|}
$$

where, $|\mathrm{I}|$ is data for the improvement of system as the scalar cardinality of fuzzy set for each of the found error. $|\mathrm{T}|$ is data for the influence of found errors on the system as the scalar cardinality of fuzzy set for each of the error. $\mathrm{Y}$ is the set of probable errors and $\mathrm{X}$ is the set of found errors. $\|\mathrm{I}\|$ is the relative size of Fuzzy set for each of the found error and $\|\mathrm{T}\|$ is the relative size of Fuzzy set for each of the error.

$$
\alpha=\frac{\sum\|T\|}{|Y|}, \beta=\frac{\sum\|I\|}{|X|}, \gamma=\frac{\|T\|}{F N}
$$


where, $\alpha, \alpha$-cut set in fuzzy set, is data for error types and functions which should be considered carefully for improvement. $\beta$ is data for system stability as the relative cardinality of fuzzy set that found errors are elements of predefined error types. $\gamma$ is the ratio of application software errors(error types $e, f$, and $g$ ) to functions of the system as the relative size of fuzzy set for error types e, $f$ and $g$. FN is the total number of functions of the system.

The parameters found in the previous step can be used in various analyses. In this paper, the parameters are used in the following 5 kinds of analyses :

Analysis of scalar cardinality distribution for each error type. $\Rightarrow>$ This shows the degree of improvement for each version upgrade.

- Analysis of relative cardinality distribution for each error type. $\Rightarrow$ This shows the functions that should be considered carefully in the next improvement and the error types that have strong influence on the system.

- Analysis of found error distribution for each improvement or software version. $\Rightarrow$ This can be used to tell the influence of errors on the degree of improvement.

- Analysis of found error distribution for each function of application software. $=>$ This tells not only the degree of implementation for each function but also is very important because the improvements of software are continued after the freezing of hardware

- Analysis of system stability for each software version. => System stability is analyzed using relative cardinality of fuzzy set comprised of fuzzy values for error types. The result can also be used as the basis for the evaluation of $R \& D$ results.

\section{APPLICATION}

The analysis method, implemented as XTAM, is applied to the ATM switching system which is under development by ETRI, Korea. Raw data are gathered from tests of several software versions for the switching system. Software versions are named as V1, V2, and V3 for convenience.

Raw data, needed for possibility distribution function, are acquired with the experience and the help of system engineering expert. The analyses of raw data give the results in Figures 1, 2, and 3. Figure 1 represents the degree of improvement for each error type with software versions. In the Figure, error types $a, b, c, d$, and e are less frequent with software versions while other error types are not improved. The trend of error type g may be explained the instability of the software. Figure 3 (b) shows the decrease of total errors. However, this does not mean that error of every type is less frequent as shown by Figure 1.

Figure 2 shows the relative cardinality distribution which shows the possibility for each error type. $\alpha$ value represents the what should be carefully debugged for improvement as mentioned before. Since $\alpha$ values are $0.23,0.25$ and 0.24 for V1, V2, and V3, respectively, efforts should be given to the error types of hardware, administration and maintenance for $\mathrm{V} 1$ and V2 and to the error types of hardware, firmware, administration, and maintenance for V3. From this it can be said that errors in maintenance application part are most influential.

Figure 3 (a) shows error distribution for each function group. The value is the ratio of scalar cardinality to the number of needed functions. The figure shows highest error ratio in the 
implementation of administration functions for V1 and V2 while shows highest error ratio in the implementation of administration functions for V3. This fact is a very important information in the improvement of software versions.

Figure 3 (b) shows the influence of errors on the system stability. The value remains nearly constant irrespective of error numbers and extent of improvement for each error type compared with that in Figures 1 and 2. This shows the fact that the errors are critical or appears in several types of errors. Therefore it may be said that the software package is not stable. The stability of a system depends on the degree of influence caused by errors.

The above results can be summarized that most errors come from hardware, maintenance and administration functions and the number and types of error decreases as the upgrade of system.

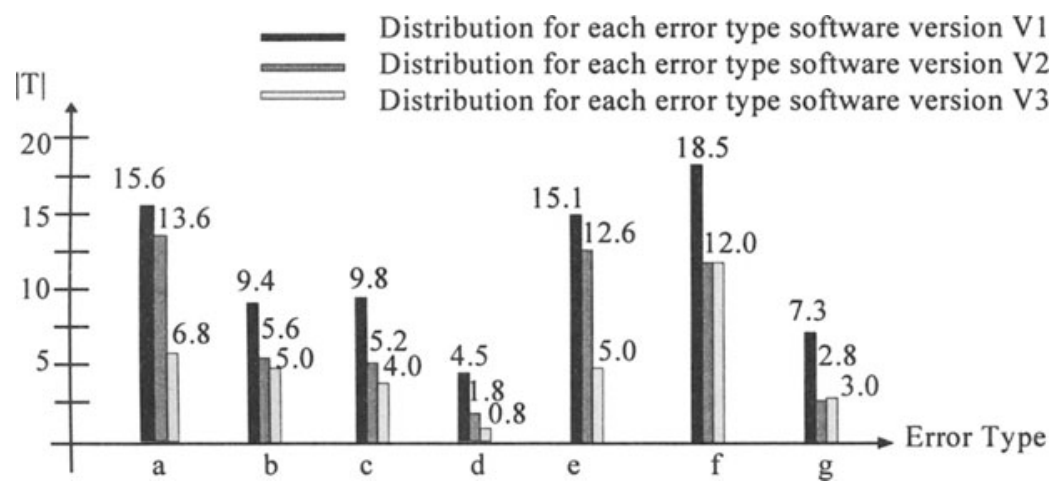

Figure 1 Scalar cardinality distribution for each error type

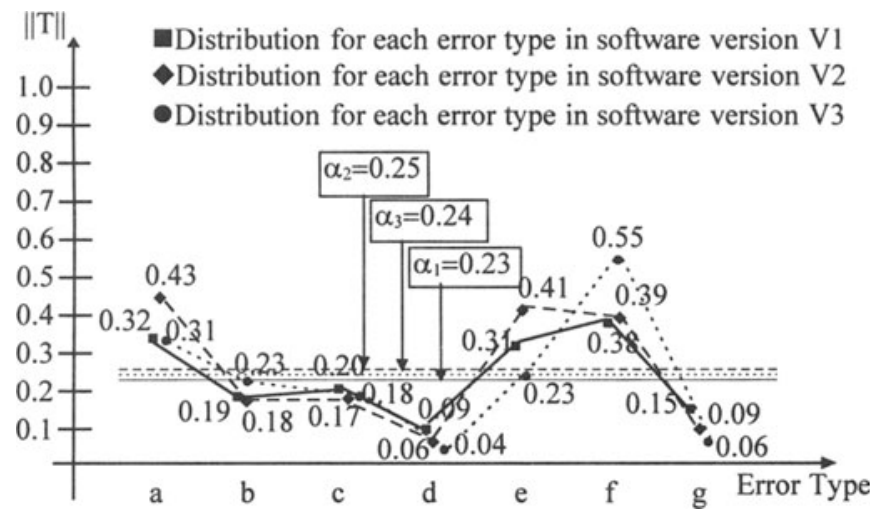

Figure 2 Relative cardinality distribution for each error type 


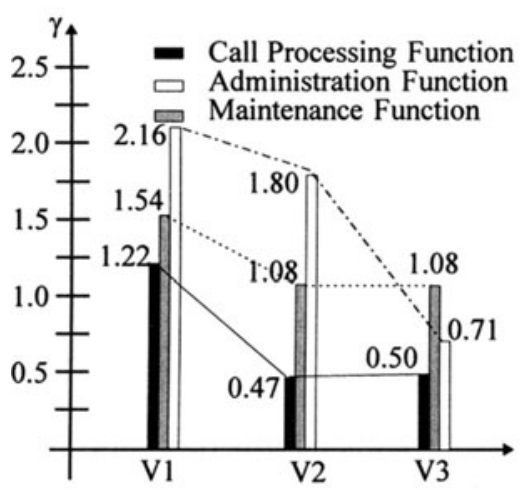

(a)

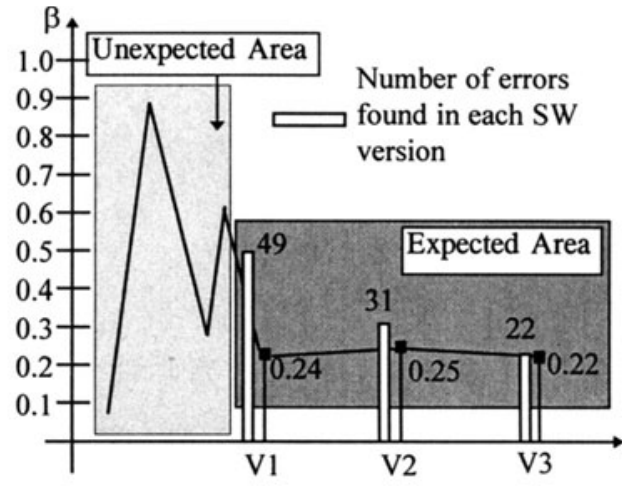

(b)

Figure 3 Error distribution for each application software of ATM Exchange and the relation with between number of errors found and system instability distribution in software version

\section{CONCLUSION}

This paper proposes a new method, called XTAM(eXchange system Test result Analysis Method) to analyze system test result. The new method has been applied for the analysis of test result for ATM switching system. By the application of new analysis method, it is possible to know the degree of system improvement, to determine parts that need careful consideration in the improvement of the system, to know the extent of implementation of functions, and to know the influence of errors for each type on the system and the stability of the system. Thus, the application of the new analysis method, XTAM, will make it easier to assure the quality of switching systems.

Since XTAM is based on possibility distribution function, correct raw data is required for correct analysis. After the correct analysis of errors found during tests by experts, it is possible to get possibility distribution function.

\section{[References]}

[1] Martin L. Katcher, "Integration and System Testing: a Methodology," ISS Session 14 C Paper 1, pp1-5, 7-11 May 1984.

[2] S.K.Cheong, S.K.Noh, and T.W.Jeong, "Testing Evaluation Method of ATM Switching System,"Analysis of Trend in Electronics and Telecom. vol.9 no.4, pp1-7, Jan.1995, ETRI

[3] S.K. Cheong, K.H. Lee, and T.W.Jeong, "On the testing of TDX-10 SSP System," Analysis of Trend in Electronics and Telecom. vol.14 no.2, pp1-7, July 1992, ETRI

[4] William E. Howden, "Errors, Design Properties and Functional Program Tests," B.Chandrasekaran and S.Radicchi, COMPUTER PROGRAM TESTING, North Holland, 
pp1 15-127,1981.

[5] Lee J.White, "Basic Mathematical Definitions and Results in Testing," B.Chandrasekaran and S.Radicchi, COMPUTER PROGRAM TESTING, North Holland, pp.13-24,1981.

[6] Timothy A. Budd, "Mutation Analysis : Ideas, Examples, Problems and Prospects," B.Chandrasekaran and S.Radicchi, COMPUTER PROGRAM TESTING, North Holland, pp129-148,1981.

[7] Leon Osterweil, “A STRATEGY FOR INTEGRATING PROGRAM TESTING AND ANALYSIS", B.Chandrasekaran and S.Radicchi, COMPUTER PROGRAM TESTING, North Holland, pp187-229,1981.

\section{[BIOGRAPHY]}

Seung-Kook Cheong was born in Korea in 1959. He received the B.S in electronic engineering from Taejeon Open National University, Korea, in 1982, and M.S in electronic engineering from Hannam University, Korea, in 1992. Since 1985, he has worked for Switching Technology Division of ETRI, as a senior researcher, pursuing R\&D on the switching systems for PSTN, N-ISDN, IN, and B-ISDN. His interested research parts are switching systems engineering for public network, ATM network design, and its evaluation.

Kyeong-Ho Lee* was born in Korea in 1956. He received the B.S and M.S in electronic engineering from Kwangwun University, Korea, in 1980, and 1982, respectively. Since 1982, he has worked for Switching Technology Division of ETRI, pursuing R\&D on the switching systems for PSTN, N-ISDN, IN, and B-ISDN. Currently, he is the head of Quality Assurance Section. His research interests include network design, telecommunication modeling and its performance evaluation.

Taeg-Won Jeong* was born in Korea in 1955. He received the B.S and M.S in electrical engineering from Seoul National University, Korea, in 1979, and 1981, respectively, and Ph.D. in electrical engineering from University of Florida, USA, in 1991. Since 1983, he has worked for ETRI, pursuing R\&D on the switching systems for PSTN, N-ISDN, IN, and B-ISDN. Currently, he is the head of ATM Service Section. 